\title{
The first complete plastid genomes of Melastomataceae are highly structurally conserved
}

\author{
Marcelo Reginato ${ }^{\text {Corresp., }}{ }^{1}$, Kurt M Neubig ${ }^{2}$, Lucas C Majure ${ }^{3}{ }^{,}$Fabian A Michelangeli ${ }^{1}$ \\ 1 Institute of Systematic Botany, The New York Botanical Garden, Bronx, New York, United States \\ 2 Department of Plant Biology, University of Carbondale, Carbondale, Illinois, United States \\ Department of Research, Conservation and Collections, Desert Botanical Garden, Phoenix, Arizona, United States \\ Corresponding Author: Marcelo Reginato \\ Email address: reginatobio@yahoo.com.br
}

Background. In the past three decades, several studies have predominantly relied on a small sample of the plastome to infer deep phylogenetic relationships in the species-rich Melastomataceae. Here, we report the first full plastid sequences of this family, compare general features of the sampled plastomes to other sequenced Myrtales, and survey the plastomes for highly informative regions for phylogenetics.

Methods. Genome skimming was performed for 16 species spread across the Melastomataceae. Plastomes were assembled, annotated and compared to eight sequenced plastids in the Myrtales. Phylogenetic inference was performed using Maximum Likelihood on six different data sets, where putative biases were taken into account. Summary statistics were generated for all introns and intergenic spacers with suitable size for PCR amplification and used to rank the markers by phylogenetic information.

Results. The majority of the plastomes sampled are conserved in gene content and order, as well as in sequence length and GC content within plastid regions and sequence classes. Departures include the putative presence of rps16 and rp/2 pseudogenes in some plastomes. Phylogenetic analyses of the majority of the schemes analyzed resulted in the same topology with high values of bootstrap support. Although there is still uncertainty in some relationships, in the highest supported topologies only two nodes received bootstrap values lower than $95 \%$.

Discussion. Melastomataceae plastomes are no exception for the general patterns observed in the genomic structure of land plant chloroplasts, being highly conserved and structurally similar to most other Myrtales. Despite the fact that the full plastome phylogeny shares most of the clades with the previously widely used and reduced data set, some changes are still observed and bootstrap support is higher. The plastome data set presented here is a step towards phylogenomic analyses in the Melastomataceae and will be a useful resource for future studies. 
1 The first complete plastid genomes of Melastomataceae are highly structurally conserved

2 Marcelo Reginato ${ }^{1}$, Kurt M. Neubig ${ }^{2}$, Lucas C. Majure ${ }^{3}$, Fabian A. Michelangeli ${ }^{1}$

$3{ }^{1}$ Institute of Systematic Botany, The New York Botanical Garden, Bronx, NY, USA

$4{ }^{2}$ Dept. of Plant Biology, Southern Illinois University of Carbondale, Carbondale, Illinois 62901

$5 \quad{ }^{3}$ Dept. of Research, Conservation and Collections, Desert Botanical Garden, Phoenix, AZ, USA

7 Corresponding author:

8 Marcelo Reginato ${ }^{1}$

9

10 Email address: reginatobio@yahoo.com.br 


\section{Abstract}

13 Background. In the past three decades, several studies have predominantly relied on a small

14 sample of the plastome to infer deep phylogenetic relationships in the species-rich

Melastomataceae. Here, we report the first full plastid sequences of this family, compare general

features of the sampled plastomes to other sequenced Myrtales, and survey the plastomes for

highly informative regions for phylogenetics.

Methods. Genome skimming was performed for 16 species spread across the Melastomataceae.

Plastomes were assembled, annotated and compared to eight sequenced plastids in the Myrtales.

Phylogenetic inference was performed using Maximum Likelihood on six different data sets,

where putative biases were taken into account. Summary statistics were generated for all introns

and intergenic spacers with suitable size for PCR amplification and used to rank the markers by phylogenetic information.

Results. The majority of the plastomes sampled are conserved in gene content and order, as well as in sequence length and GC content within plastid regions and sequence classes. Departures include the putative presence of rps 16 and $r p l 2$ pseudogenes in some plastomes. Phylogenetic analyses of the majority of the schemes analyzed resulted in the same topology with high values of bootstrap support. Although there is still uncertainty in some relationships, in the highest supported topologies only two nodes received bootstrap values lower than $95 \%$.

Discussion. Melastomataceae plastomes are no exception for the general patterns observed in the genomic structure of land plant chloroplasts, being highly conserved and structurally similar to most other Myrtales. Despite the fact that the full plastome phylogeny shares most of the clades with the previously widely used and reduced data set, some changes are still observed and 
34 bootstrap support is higher. The plastome data set presented here is a step towards phylogenomic

35

36

37

38

39

40

41

42

43

44

45

46

47

48

49

50

51

52

53

54

55

56 analyses in the Melastomataceae and will be a useful resource for future studies.

Keywords Chloroplast, Melastomataceae, Myrtales, NGS, phylogenomics, plastome, genome skimming

\section{Introduction}

The Melastomataceae Juss. has over 5000 species distributed predominantly across the tropical regions. The observed levels of diversity, endemism or abundance of its members across different habitats make the family an important ecological group, as well as an excellent model for a variety evolutionary studies. The Melastomataceae belong in the Myrtales, where it is sister to the small CAP clade (Crypteroniaceae, Alzateaceae and Penaeaceae), which all together form a clade sister to Myrtaceae + Vochysiaceae (Berger et al., 2015). Plastid markers along with the nuclear ribosomal spacers (nrETS and nrITS) have been the major, and very often the exclusive, source of phylogenetic information in the family. Melastomataceae debut in molecular phylogenies was in a Myrtales-focused study, based on a partial amino acid sequence of the $r b c S$ gene (Martin \& Dowd, 1986). This study was followed by a more comprehensive nucleotidebased phylogeny, where the plastid $r b c L$ gene was analyzed (Conti, Litt \& Systma, 1996). The first Melastomataceae-wide phylogeny used a plastid data set including the $r b c L$ and $n d h F$ genes plus the rpl16 intron (Clausing \& Renner, 2001). This plastid data set is still the most employed source of information in studies focusing on generic relationships across the family (Fritsch et al., 2004; Renner, 2004; Amorim, Goldenberg \& Michelangeli, 2009; Michelangeli et al., 2011; Goldenberg et al., 2012; Michelangeli, Ulloa \& Sosa, 2014; Goldenberg et al., 2015; Zeng et al., 
57 2016). Phylogenetic studies within lower lineages of Melastomataceae have predominantly used

58 the plastid spacers $a c c D$-psaI, atpF-atpH, psbK-psbI, and trnS-trnG, along with the ribosomal

59 spacers nrETS and nrITS (Bécquer-Granados et al., 2008; Reginato, Michelangeli \&

60 Goldenberg, 2010; Kriebel, Michelangeli \& Kelly, 2015, Reginato \& Michelangeli, 2016).

61 Recently, the latter data set has also been used at deeper level studies (Michelangeli et al., 2013;

62 Rocha et al., 2016).

Family-wide phylogenetic studies based on plastid markers have uncovered major

relationships in the Melastomataceae, with several implications to the classification and evolutionary understanding in the family. Early studies have consolidated the sister relationship of Olisbeoideae and the remaining Melastomataceae, settling on the currently accepted family circumscription (Conti, Litt \& Systma, 1996; APG, 1998; but see Clausing \& Renner, 2001 for a different perspective). Latter studies focused in some tribal re-arrangements (Fritsch et al., 2004; Penneys et al., 2010, Michelangeli et al., 2011), generic placement (Amorim, Goldenberg \& Michelangeli, 2009; Goldenberg et al., 2012; Michelangeli, Ulloa \& Sosa, 2014; Goldenberg et al., 2015; Kriebel, 2016; Rocha et al., 2016; Zeng et al., 2016), phylogenetic evaluation of higher species-rich lineages (Michelangeli et al., 2004, Stone, 2006; Goldenberg et al., 2008; Martin et al., 2008; Michelangeli et al., 2008; Michelangeli et al., 2013), and lower taxon phylogenies (Bécquer-Granados et al., 2008; Reginato, Michelangeli \& Goldenberg, 2010; Penneys, 2013; Kriebel, Michelangeli \& Kelly, 2015; Gamba-Moreno \& Almeda, 2014; Majure et al., 2015; Reginato \& Michelangeli, 2016). Even in family-wide phylogenies, the level of variation across these few sampled plastid markers is unsatisfactory, as evidenced by low statistical support among many relationships in different published analyses. This issue becomes more prominent in phylogenetic analyses of lineages within Melastomataceae, where the plastid phylogeny is 
80

81

82

83

84

85

86

87

88

89

overall weakly supported, and concatenated results tend to be dominated by the more variable nuclear ribosomal data (Reginato, Michelangeli \& Goldenberg, 2010; Reginato \& Michelangeli 2016).

Phylogenomic studies are sparse in the Myrtales and absent in the Melastomataceae.

Currently, there are 54 full plastids of Myrtales on the NCBI database, covering three out of the nine families in the order (Lythraceae, Myrtaceae and Onagraceae). Full plastomes can potentially improve hypotheses of phylogenetic relationships within the family, as well as in the Myrtales, and provide basic information for other aspects of molecular biology (e.g., DNA barcoding, plastome evolution, development of molecular markers). Here, we present the first complete plastid genomes in the Melastomataceae, covering 16 species spread across the family. The objectives of this study are to describe the structure of the sampled plastomes; compare main features of the plastomes within the family and to other available Myrtales plastomes; and survey the plastomes for highly informative phylogenetic markers for future use.

\section{Material and methods}

\section{Taxon sampling, DNA extraction and sequencing}

Genome skimming was performed for 16 species of Melastomataceae. Sampling was based on previous family wide phylogenetic studies (Michelangeli et al., 2014; Goldenberg et al., 2015), where each sample belongs to a different major lineage of the family, either with a formal tribe status or not. Voucher information along with GenBank accession codes are presented in Table 1. Total genomic DNA was isolated from silica-dried tissue using the Qiagen DNAeasy plant mini-kit (Qiagen, Valencia, CA) following the protocol suggested by Alexander et al. (2007) or used a modified CTAB extraction where the aqueous supernatant was silica-column 
103 purified (Neubig et al., 2014). Total DNA samples were quantified using a NanoDrop

104 Spectrophotometer (Thermo Scientific, Waltham, MA, USA) or Qubit 2.0 (Invitrogen, Carlsbad,

105 CA, USA). Total genomic libraries and barcoding was performed at Cold Spring Harbor

106 Laboratories or at Rapid Genomics (Gainesville, FL) for sequencing on an Illumina HiSeq2000

107 platform (Illumina, Inc., San Diego, CA, USA).

108

109

110

111

112

113

114

115

116

117

118

119

120

121

122

123

124

\section{Plastid genome assembly and annotation}

Total reads number yielded was on average ca. $11.5 \mathrm{~Gb}$ per sample (s.d. $=6 \mathrm{~Gb}$ ). Paired reads were imported into Geneious 7.1 (Biomatters Ltd., Auckland, New Zealand), trimmed by quality (at 0.05 probability) and de novo assembled (Geneious Assembler, "low sensitivity" option, default settings). Filtered assembled contigs (length $>1 \mathrm{~kb}$ ) were blasted against the Eucalyptus polybractea plastome (NC022393). The identified plastid contigs were then reference assembled against the E. polybractea plastome in order to generate a single contig to construct the circular maps. Eventual short gaps were filled by iteratively mapping the total paired reads against the contig ends. Plastid annotation was performed in Geneious 7.1 with Arabidopsis thaliana (NC000932) and Eucalyptus polybractea (NC022393) as references. Graphical representations of the plastid circular and linear maps were generated with OGDRAW (Lohse et al., 2013) and the R package genoPlotR (R Core Team, 2016; Guy, Kultima \& Andersson, 2010).

Plastome structure, gene content, and general characteristics of the plastid genome were compared among the 16 Melastomataceae plastomes and to eight published plastomes of Myrtales, covering all families in this order available on the NCBI website. The Myrtales plastomes included one species in the Lythraceae (Lagerstroemia fauriei - NC029808), one 
Onagraceae (Oenothera grandiflora - NC029211) and six Myrtaceae (Allosyncarpia ternata -

126 NC022413; Angophora costata - NC022412; Corymbia gummifera - NC022407; Eucalyptus

127 polybractea - NC022393; Eugenia uniflora - NC027744; and Stockwellia quadrifida -

128 NC022414).

129 Phylogenetic analyses

130 Three major data sets were generated for phylogenetic inference. The first included the

131 non-coding regions (ncs data set), the second included 78 protein-coding genes (cds data set),

132 and the third consisted of fully assembled plastomes (full data set). In all data sets one of the IR

133 sequences was removed to reduce overrepresentation of duplicated sequences. Full plastids were

134 aligned with MAFFT v. 7 using the FFT-NS-i x 1000 strategy (Katoh, 2013). Coding sequences

135 were extracted from the full alignment, resulting in the cds and ncs data sets. Each gene in the

136 cds data set was re-aligned using its translation under the same strategy of the full data set and

137 then concatenated. Given that phylogenetic inference might be biased by poorly aligned regions

138 with ambiguous homology, heterogeneous rates of substitution in the different codon positions,

139 synonymous substitutions in Arginine, Leucine and Serine codons, among others (Misof \&

140 Misof, 2009; Cox et al., 2014), we further divided the three major data sets into six different

141 schemes where we attempted to circumvent those issues. Poorly aligned regions of the ncs data

142 set were removed using aliscore.pl with the $-\mathrm{N}$ and $-\mathrm{r}$ options (Misof \& Misof, 2009), and in the

143 cds data set; all codons coding for Arginine, Leucine and Serine were ambiguated. Thus, the

144 final six schemes included: 1. all ncs data set (ncs); 2. ncs data set without poorly aligned sites

145 (ncs filtered); 3. all cds data set (cds); 4. cds with A, L and S codons ambiguated (cds

146 ambiguated); 5. translated cds (protein); 6. ncs filtered plus all cds non-ambiguated (full).

147 Additionally, in order to carry out a more objective comparison with previous phylogenetic 
148 hypotheses, we also analyzed a reduced data set that included only the three more commonly

149 used markers for family wide phylogenies in the Melastomataceae ( $n d h F$ and $r b c L$ genes along

150 with the rpl16 intron, concatenated).

Phylogenetic inference for all schemes was performed using Maximum Likelihood

152 implemented in RAxML 8.2.4 (Stamatakis, 2014). The GTR+G model was employed for all

153 nucleotide data and the PROT $+\mathrm{G}$ model for the protein sequences. Support was estimated

154 through 1000 bootstrap replicates. Protein-coding sequences were partitioned by codon position

155 in all schemes, while no partitioning was employed for the non-coding regions.

\section{Phylogenetic informative regions}

In order to identify and rank highly phylogenetically informative regions in the

Melastomataceae plastomes, all introns (19) and variable intergenic spacers with suitable size for PCR amplification (22) were selected and compared. Each individual marker was aligned with MAFFT (FFT-NS-i x 1000 strategy), and its Maximum likelihood tree inferred with RAxML (not partitioned, GTR $+\mathrm{G}$ model, 100 bootstrap replicates). For each marker, we report the number of variable sites, number of parsimony informative sites, mean sequence distance (under K80 model), alignment length, mean sequence length, mean bootstrap support and distance to the full scheme plastid tree (RF distance; Robinson \& Foulds, 1981). The metrics were retrieved using functions of the R packages ape and phangorn (Paradis, Claude \& Strimmer, 2004; Schliep, 2011). Markers were ranked by phylogenetic information using a weighted mean of relative values of the following metrics: number of variable sites (weight $=1$ ), mean bootstrap 168 (weight=2) and distance to the full plastid tree (weight=3). For the top 10 markers identified in the previous step, we designed primer pairs for PCR amplification. Primers flanking the target 
170 regions were designed with Primer3, using the default settings (Rozen \& Skaletsky, 2000). All

171 metrics reported, as well primer design, considered only the ingroup (the 16 Melastomataceae

172 plastids).

\section{Results}

\section{Plastome structure}

All plastomes have a quadripartite organization, with one large single copy region (LCS), one small single copy (SSC) and two inverted repeats (IRs). A circular map of the Miconia dodecandra plastome is presented in Figure 1 and linear maps of all Melastomataceae plastomes in Figure 2. Sequence depth ranged from 42 to $705($ mean $=289)$ and plastome length from 153,311 to $157,216 \mathrm{bp}($ mean $=155,806 \mathrm{pb})$. Sequence length and GC content of the different regions across the Melastomataceae plastomes are presented in Table 2. Overall, GC content is similar across species within the same plastid region, while the LSC regions has the greatest standard deviation in sequence length (s.d. $=616 \mathrm{bp}$ ), followed by IR (s.d. $=250 \mathrm{bp}$ ) and the $\operatorname{SSC}($ s.d. $=126$ bp). ribosomal (rRNA), totaling 129 genes (including duplicates and $y c f 1, y c f 2, y c f 3$ and $y c f 4$ ). regions, GC content is similar across different species within the same sequence class (CDS, tRNA, rRNA, intron and intergenic spacers), whereas the greatest variation in sequence length is observed across intergenic spacers $($ s.d. $=617 \mathrm{bp})$. A comparative summary of length and GC 
192 3. In the majority of the species sampled, gene content and order is similar to other Myrtales

193 plastids, such as Lagerstroemia fauriei (NC029808) and Eucalyptus polybractea (NC022393).

194 The exceptions are $r p s 16$ and $r p l 2$, which are putative pseudogenes in some plastids. The former

195 seems to have been pseudogenized in Graffenrieda moritziana and Pterogastra divaricata

196 (where the first exon is absent) and in Salpinga margaritacea (with several insertions changing

197 the reading frame in the second exon); the second copy of $r p l 2$ gene (in the IRB) is likely a

198 pseudogene in Salpinga margaritacea due to a shift in the IRB-LSC boundary in that plastid,

199 which resulted in the loss of the second exon. Additionally, some variation is observed in all

200 region boundaries across the Melastomataceae plastomes. The LSC-IRA boundary is located in

201 the rps19 gene in most species, except in S. margaritacea where it is located in the intron of the

$202 r p l 2$ gene; the IRA-SSC boundary is located in the overlapping $\psi y c f l$ and $n d h F$; the SSC-IRB in

203 the $y c f 1$; and the IRB-LSC in the $r p l 2$-trnH spacer or in the $t r n H$ gene. Introns are found in 17

204 genes in all Melastomataceae plastomes, including six tRNA genes and 11 protein-coding genes,

205 from which three have two introns (clpP, rps12 and $y c f 3)$. A comparison of the number of genes,

206 regions and plastome length of one Melastomataceae (M. dodecandra) and eight Myrtales

207 plastids is presented in Table 4. The sequence length of the full plastome and its regions in the

208 Melastomataceae sampled here are in the range observed for other Myrtales.

\section{Phylogenetic analyses}

The majority of the six analytical schemes recovered the same topology (Figures 2 and

211 3B). The only exception was the "all non-coding" scheme (i.e., the full non-coding regions

212 without filtering of dubiously aligned base pairs), where Blakea + Opistocentra, Triolena +

213 Merianthera and Rhynchanthera assume a different position (Figure 3A). Pairwise tree distances

214 among all schemes are depicted in Figure 3C, and all Maximum Likelihood trees with bootstrap 
215 support values are given in the Supplementary Figure S1. Bootstrap support is highest in the

216 "full" and "cds" schemes and lower in the "protein" and "all non-coding" schemes (Figure 3D).

217 In the highest supported topologies, there are only two nodes with bootstrap values lower than

21895 , and those involve the relationship disagreements between the two alternate topologies

219 (Figure 3A-B). While filtering the non-coding poorly aligned sites improved bootstrap support

220 and also changed the topology ("ncs" vs. "ncs filtered", Figure 3), ambiguating common amino

221 acids in the coding sequences did not have any apparent effect in the topology or support values

222 ("cds" vs. "cds ambiguated"; Figure 3D).

The commonly used plastid data set in previous family-wide studies ( $r b c L, n d h F$ and rpl16 intron) also resulted in a different topology from the "full" scheme, although with most clades in common (supplementary Figure S2). Disagreements involved the position of Allomaieta, Trioleta + Merianthera, Blakea + Opisthocentra, and Rhynchanthera; these disagreements manifest in nodes of low bootstrap support where, in the reduced data set, they range from 24 to $100($ mean $=73)$.

\section{Phylogenetically informative regions}

Summary statistics for all intron and intergenic spacers with suitable size for PCR amplification are presented in Supplementary Table S1. A list of the top 10 markers ranked by phylogenetic information, taking into account topological distance to the tree based on the "full" scheme (Figure 2), mean bootstrap support and number of variable sites is given in Table 5, and the full list is available in supplementary Table S1. All single marker phylogenies presented 235 some disagreement to the tree based on the "full" scheme (RF tree distance ranging from 4 to 
$237507($ mean $=224)$. Primer pair sequences for PCR amplification are provided for the top 5

238 markers in Table 6.

239

240

\section{Discussion}

Plastid genomes of higher plants are of relatively small size, ranging from 115 to $165 \mathrm{~kb}$

in most groups, with an average of 90 protein-coding genes across most land plants (Ravi et al., 2007, Wicke et al., 2011). In general, the quadripartite organization, gene content and order are conserved, and GC content is usually stable within plastid regions and sequence classes (Ravi et al., 2007, Wicke et al., 2011). Melastomataceae plastomes are no exception for these patterns, being highly conserved and structurally similar to most other Myrtales, as well as to an ordinary angiosperm plastome. Melastomataceae plastomes' mean length $(156 \mathrm{~kb})$ is closer to the upper bound observed across most plants $(165 \mathrm{~kb})$, while the number of genes and GC content are around the average (90 genes, $\mathrm{GC}=37 \%$; Ravi et al., 2007). High conservation in genomic structure of plastomes among the Myrtales has been previously suggested (Gu et al., 2016) and is extended here to include Melastomataceae. The greatest variation in sequence length among different region classes in Melastomataceae are observed in the intergenic spacers, which is also another general pattern in plastomes (Ravi et al., 2007, Gu et al., 2016). Additionally, the boundaries of the IRs vary, as observed in some Myrtales and other groups (Bayly et al., 2013). a useful tool for plant phylogenetic studies (Ravi et al. 2008). An updated comprehensive

257 phylogenetic hypothesis for the entire Melastomataceae is overdue, and full plastid sequences 258 would contribute greatly to such an endeavor. Additionally, as sampling increases in the 
260 (e.g., Combretaceae position, Berger et al., 2015). Despite the fact that the full plastome

261 phylogeny recovered here shares most of the clades with the widely used "rbcL $+n d h F+r p l 16$ "

262 tree, some changes are still observed and bootstrap support is higher. A more conclusive account

263 on the extent of such changes will require more taxa to be sampled.

264 Here, we provide a list of potentially highly informative plastid markers for

265 Melastomataceae. We acknowledge that the information descriptors employed are very sensitive

266 to the taxa under analysis. Nonetheless, this ranked list can be used as guidance for sampling

267 design of future studies, whereas the new family specific primers will increase the plastid options

268 for Sanger sequencing-based phylogenies. There has been some debate as to whether the

269 availability of full plastome sequencing (and other NGS tools) would render Sanger sequencing

270 obsolete (Hert et al., 2008). Here we show that a full plastome phylogeny is an improvement on

271 single or few plastid loci phylogenies, especially on the level of statistical support. However,

272 considering scalability, computational complexity and budget limitations, a comprehensive

273 NGS-based phylogeny for the mega-diverse Melastomataceae might not be achieved in the short

274 term. Nonetheless, an expanded full plastome data set along with the more abundant Sanger-

275 based sequences available, could be coupled in future studies. A hybrid NGS and Sanger

276 sequencing approach has been employed for other groups (Xi et al., 2012; Leaché et al. 2014;

277 Gardner et al., 2016), and could help clarifying the backbone of a comprehensive

278 Melastomataceae phylogeny. Recalcitrant phylogenetic backbones are a widespread and

279 challenging phenomenon in angiosperms (Xi et al., 2012; Straub et al., 2014), and their

280 resolution is critical to increase the confidence of ancestral state reconstructions, historical

281 biogeographical scenarios and other evolutionary hypotheses. Although full plastomes, or an

282 expanded sample of plastid markers, may help to improve the confidence of phylogenetic 
283 relationships within the Melastomataceae, we also recognize the need of parallel sampling of

284 additional independent genealogies (i.e., nuclear and mitochondrial genomes) for further

285 refinement in the Melastomataceae tree.

286

287 Acknowledgments

288 We thank all collaborators who kindly provided tissue samples for this study. We also 289 thank Kenneth Karol for helpful insights on assembling procedures. We thank Rapid Genomics

290 (Gainesville, FL) for sequencing services.

291

292

\section{Funding}

293

This study was supported by the National Science Foundation (DEB-0818399 and DEB-

294

1343612).

295

296

References

297

298

299

300

301

302

Alexander PJ, Rajanikanth G, Bacon CD, Bailey CD. 2007. Rapid inexpensive recovery of high quality plant DNA using a reciprocating saw and silica-based columns. Molecular Ecology Notes 7: 5-9.

Amorim AM, Goldenberg R, Michelangeli FA. 2009. A new species of Physeterostemon (Melastomataceae) from Bahia, Brazil, with notes on the phylogeny of the genus. Systematic Botany 34: 324-329. 
303 304

APG (Angiosperm Phylogeny Group). 1998. An ordinal classification for the families of flowering plants. Annals of the Missouri Botanical Garden 85: 531-553.

Bayly MJ, Rigault P, Spokevicius A, Ladiges PY, Ades PK, Anderson C, Bossinger G, Merchant A, Udovicic F, Woodrow IE, Tibbits J. 2013. Chloroplast genome analysis of Australian eucalypts-Eucalyptus, Corymbia, Angophora, Allosyncarpia and Stockwellia (Myrtaceae). Molecular Phylogenetics and Evolution 69: 704-716.

Bécquer-Granados ER, Neubig KM, Judd WS, Michelangeli FA, Abbott JR, Penneys DS. 2008. Preliminary molecular phylogenetic studies in Pachyanthus (Miconieae, Melastomataceae). The Botanical Review 74: 37-52.

Berger BA, Kriebel R, Spalink D, Sytsma KJ. 2016. Divergence times, historical biogeography, and shifts in speciation rates of Myrtales. Molecular Phylogenetics and Evolution 95: 116-136.

Clausing G, Renner SS. 2001. Molecular phylogenetics of Melastomataceae and Memecylaceae: implications for character evolution. American Journal of Botany 88: 486-498.

Conti E, Litt A, Sytsma KJ. 1996. Circumscription of Myrtales and their relationships to other rosids: evidence from $r b c L$ sequence data. American Journal of Botany 83: 221-233.

Cox CJ, Li B, Foster PG, Embley TM, Civáň P. 2014. Conflicting phylogenies for early land plants are caused by composition biases among synonymous substitutions. Systematic Biology 63: 272-279. 
323 Fritsch PW, Almeda F, Renner SS, Martins AB, Cruz BC. 2004. Phylogeny and

324 circumscription of the near-endemic Brazilian tribe Microlicieae (Melastomataceae). American Journal of Botany 91: 1105-1114.

326

327

Gamba-Moreno DL, Almeda F. 2014. Systematics of the Octopleura clade of Miconia (Melastomataceae: Miconieae) in tropical America. Phytotaxa 179: 1-174.

Gardner AG, Sessa EB, Michener P, Johnson E, Shepherd KA, Howarth DG, Jabaily RS. 2016. Utilizing next-generation sequencing to resolve the backbone of the Core Goodeniaceae and inform future taxonomic and floral form studies. Molecular Phylogenetics and Evolution 94: 605-617.

Goldenberg R, Penneys DS, Almeda F, Judd WS, Michelangeli FA. 2008. Phylogeny of Miconia (Melastomataceae): patterns of stamen diversification in a megadiverse neotropical genus. International Journal of Plant Sciences 169: 963-979.

Goldenberg R, Fraga CN, Fontana AP, Nicolas AN, Michelangeli FA. 2012. Taxonomy and phylogeny of Merianthera (Melastomataceae). Taxon 61: 1040-1056.

\section{Goldenberg R, Almeda F, Sosa K, Ribeiro RC, Michelangeli FA. 2015. Rupestrea: A new} Brazilian genus of Melastomataceae, with anomalous seeds and dry indehiscent fruits. Systematic Botany 40: 561-571.

Gu C, Tembrock LR, Johnson NG, Simmons MP, Wu Z. 2016. The complete plastid genome of Lagerstroemia fauriei and loss of rpl2 intron from Lagerstroemia (Lythraceae). PloS One 11: e0150752. 
343 Guy L, Kultima JR, Andersson SG. 2010. genoPlotR: comparative gene and genome

344 visualization in R. Bioinformatics 26: 2334-2335.

345 Hert DG, Fredlake CP, Barron AE. 2008. Advantages and limitations of next-generation sequencing technologies: A comparison of electrophoresis and non-electrophoresis methods. Electrophoresis 29: 4618-4626.

348

Katoh S. 2013. MAFFT multiple sequence alignment software version 7: improvements in performance and usability. Molecular Biology and Evolution 30: 772-780.

Kriebel R, Michelangeli FA, Kelly LM. 2015. Discovery of unusual anatomical and continuous characters in the evolutionary history of Conostegia (Miconieae: Melastomataceae). Molecular Phylogenetics and Evolution 82: 289-313.

Kriebel R. 2016. Phylogenetic placement of the monotypic genus Schwackaea (Melastomeae: Melastomataceae) and the evolution of its unique fruit. International Journal of Plant Sciences 177: 440-448.

Leaché, AD, Wagner P, Linkem CW, Böhme W, Papenfuss TJ, Chong RA, Lavin BR, Bauer AM, Nielsen SV, Greenbaum E, Rödel M, Schmitz A, LeBreton M, Ineich I, Chirio L, Ofori-Boateng C, Eniang EA, El Din SB, Lemmon AR, Burbrink FT. 2014. A hybrid phylogenetic-phylogenomic approach for species tree estimation in African Agama lizards with applications to biogeography, character evolution, and diversification. Molecular Phylogenetics and Evolution 79: 215-230. 
362 Lohse M, Drechsel O, Kahlau S, Bock R. 2013. Organellar Genome DRAW—a suite of tools

363 for generating physical maps of plastid and mitochondrial genomes and visualizing

364 expression data sets. Nucleic Acids Research 41: W575-W581.

Majure LC, Neubig KM, Skean JD, Bécquer-Granados ER, Judd WS. 2015. Evolution of the Sandpaper clade (Miconieae, Melastomataceae). International Journal of Plant Sciences 176: 607-626.

Martin CV, Little DP, Goldenberg R, Michelangeli FA. 2008. A phylogenetic evaluation of Leandra (Miconieae, Melastomataceae): a polyphyletic genus where the seeds tell the story, not the petals. Cladistics 24: 315-327.

Martin PG, Dowd JM. 1986. Phylogenetic studies using protein sequences within the order Myrtales. Annals of the Missouri Botanical Garden 73: 442-448.

Michelangeli FA, Penneys DS, Giza J, Soltis D, Hils MH, Skean JD. 2004. A preliminary phylogeny of the tribe Miconieae (Melastomataceae) based on nrITS sequence data and its implications on inflorescence position. Taxon 53: 279-279.

Michelangeli FA, Judd WS, Penneys DS, Skean JD, Bécquer-Granados ER, Goldenberg R, Martin CV. 2008. Multiple events of dispersal and radiation of the tribe Miconieae (Melastomataceae) in the Caribbean. The Botanical Review 74: 53-77.

Michelangeli FA, Nicolas A, Morales ME, David H. 2011. Phylogenetic relationships of Allomaieta, Alloneuron, Cyphostyla, and Wurdastom (Melastomataceae) and the resurrection of the tribe Cyphostyleae. International Journal of Plant Sciences 172: $1165-1178$. 
383 Michelangeli FA, Guimarães PJ, Penneys DS, Almeda F, Kriebel R. 2013. Phylogenetic

384 relationships and distribution of new world Melastomeae (Melastomataceae). Botanical Journal of the Linnean Society 171: 38-60.

386

387

Michelangeli FA, Ulloa CU, Sosa K. 2014. Quipuanthus, a new genus of Melastomataceae from the foothills of the Andes in Ecuador and Peru. Systematic Botany 39: 533-540.

Misof B, Misof K. 2009. A Monte Carlo approach successfully identifies randomness in multiple sequence alignments: a more objective means of data exclusion. Systematic Biology 58: 21-34.

Neubig KM, Whitten WM, Abbott JR, Elliott S, Soltis DE, Soltis PS. 2014. Variables affecting DNA preservation in archival DNA specimens. In: Applequist WL, Campbell LM, eds. DNA banking in the $21^{\text {st }}$ Century: Proceedings of the U.S. workshop on DNA banking. St. Louis: The William L. Brown Center at the Missouri Botanical Garden.

Paradis E, Claude J, Strimmer K. 2004. APE: analyses of phylogenetics and evolution in R language. Bioinformatics 20: 289-290.

Penneys DS, Michelangeli FA, Judd WS, Almeda F. 2010. Henrietteeae (Melastomataceae): a new neotropical berry-fruited tribe. Systematic Botany 35: 783-800.

Penneys DS. 2013. Preliminary phylogeny of the Astronieae (Melastomataceae) based on nuclear and plastid DNA sequence data, with comments on the Philippine endemic genus, Astrocalyx. Philippine Journal of Science 142: 159-168.

R Core Team. 2016. $R$ : A language and environment for statistical computing. $R$ Foundation for Statistical Computing. Vienna, Austria. http://www.R-project.org/. 
404 Ravi V, Khurana JP, Tyagi AK, Khurana P. 2008. An update on chloroplast genomes. Plant $405 \quad$ Systematics and Evolution 271: 101-122.

406

407

408

409

410

411

412

413

414

415

416

417

418

419

420

421

422

423

Reginato M, Michelangeli FA, Goldenberg R. 2010. Phylogeny of Pleiochiton

(Melastomataceae, Miconieae): total evidence. Botanical Journal of the Linnean Society 162: 423-434.

Reginato M, Michelangeli FA. 2016. Untangling the phylogeny of Leandra s. str. (Melastomataceae, Miconieae). Molecular Phylogenetics and Evolution 96: 17-32.

Renner SS. 2004. Bayesian analysis of combined chloroplast loci, using multiple calibrations, supports the recent arrival of Melastomataceae in Africa and Madagascar. American Journal of Botany 91: 1427-1435.

Robinson D, Foulds LR. 1981. Comparison of phylogenetic trees. Mathematical Biosciences 53: $131-47$.

Rocha MJR, Guimarães PJF, Michelangeli FA, Romero R. 2016. Phylogenetic placement and a new circumscription of Poteranthera (Microlicieae; Melastomataceae). Phytotaxa 263: 219-232.

Rozen S, Skaletsky H. 2000. Primer3 on the $W W W$ for general users and for biologist programmers. In: Misener S, Krawetz SA, eds. Methods in Molecular Biology 132: Bioinformatics methods and protocols, 365-386. New Jersey: Humana Press, Totowa, USA.

Schliep KP. 2011. phangorn: phylogenetic analysis in R. Bioinformatics 27: 592-593. 
424 Stamatakis A. 2014. RAxML Version 8: a tool for phylogenetic analysis and post-analysis of 425 large phylogenies. Bioinformatics 30: 1312-1313.

426

427

428

429

430

431

432

433

434

435

436

437

438

439

440

441

442

Stone RD. 2006. Phylogeny of major lineages in Melastomataceae, subfamily Olisbeoideae: utility of nuclear glyceraldehyde 3-phosphate dehydrogenase (GapC) gene sequences. Systematic Botany 31: 107-121.

Straub SC, Moore MJ, Soltis PS, Soltis DE, Liston A, Livshultz T. 2014. Phylogenetic signal detection from an ancient rapid radiation: Effects of noise reduction, long-branch attraction, and model selection in crown clade Apocynaceae. Molecular Phylogenetics and Evolution 80: 169-185.

Wicke S, Schneeweiss GM, Müller KF, Quandt D. 2011. The evolution of the plastid chromosome in land plants: gene content, gene order, gene function. Plant Molecular Biology 76: 273-297.

Xi Z, Ruhfel BR, Schaefer H, Amorim AM, Sugumaran M, Wurdack KJ, Endress PK, Matthews ML, Stevens PF, Mathews S, Davis CC. 2012. Phylogenomics and a posteriori data partitioning resolve the Cretaceous angiosperm radiation Malpighiales. Proceedings of the National Academy of Sciences 109: 17519-17524.

Zeng SJ, Zou LH, Wang P, Hong WJ, Zhang GQ, Chen LJ, Zhuang XY. 2016. Preliminary phylogeny of Fordiophyton (Melastomataceae), with the description of two new species. Phytotaxa 247: 45-61. 
Figure 1

Figure 1

Map of the Miconia dodecandra plastid genome. Genes shown outside the outer circle are transcribed clockwise and genes inside the outer circle are transcribed counterclockwise.

Genes in different functional groups are color coded following the legend. The shaded area inside the inner circle indicates the GC content, with dark shading indicating percent CG.

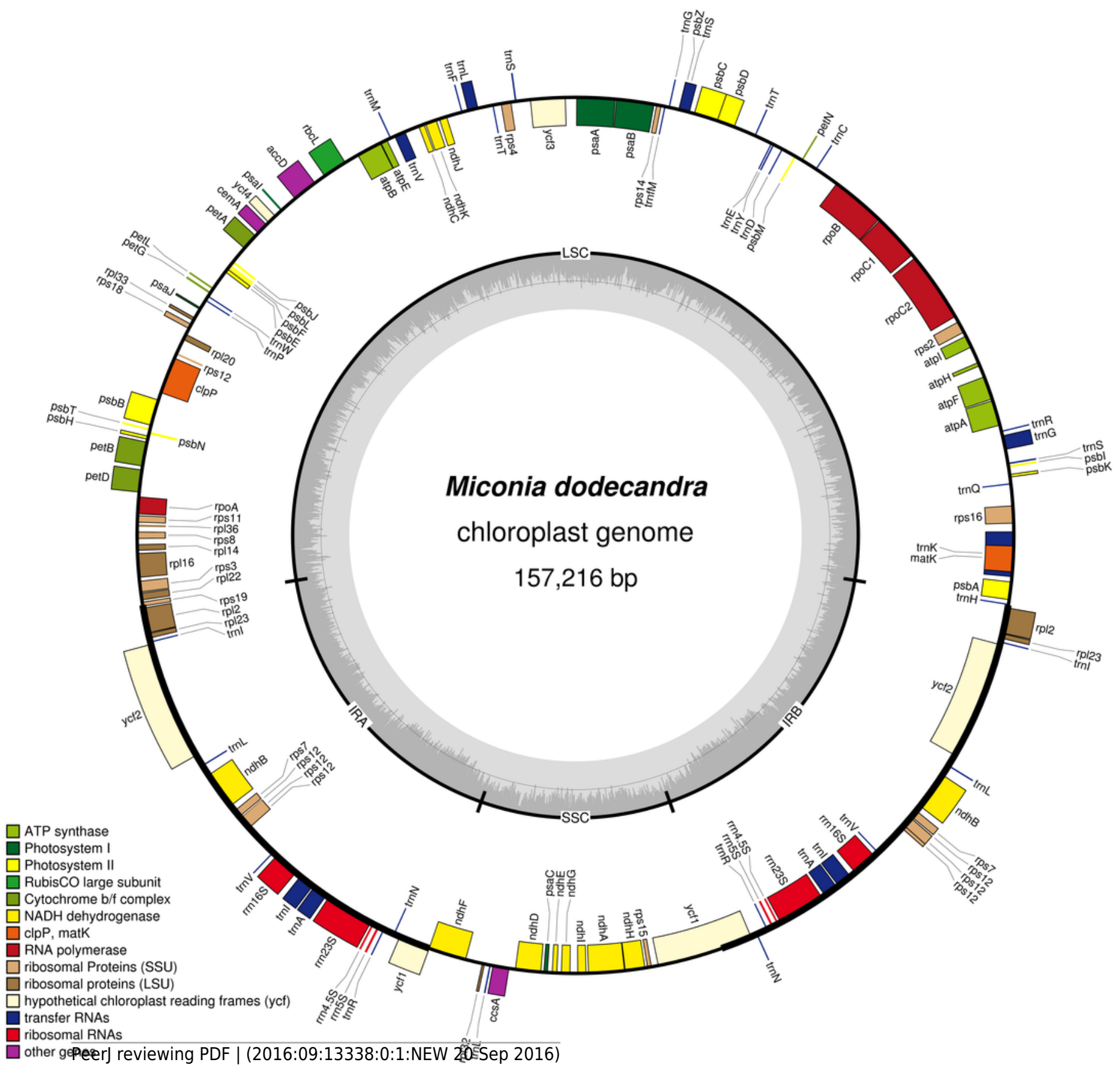




\section{Figure 2}

Figure 2

Maximum likelihood tree recovered with the full data set (left). On the right, linear plastid maps of the 16 Melastomataceae species. All genes are depicted as arrows (indicating transcription direction) and color coded following the legend of Figure 1. Gray lines link the same genes on contiguous maps. LSC (long single copy region); SSC (small single copy region); IRA (inverted repeat A); IRB (inverted repeat $B$ ).
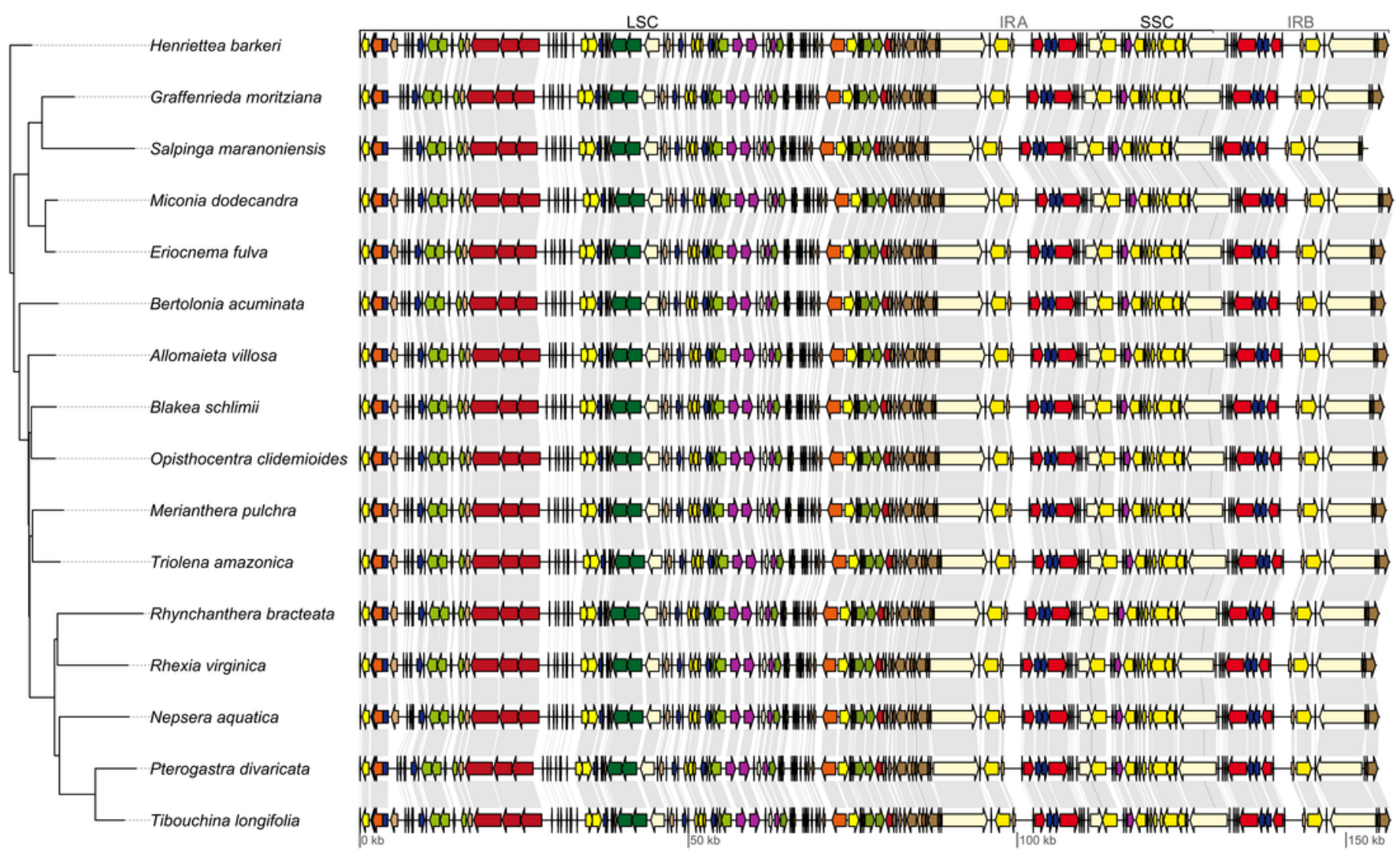


\section{Figure 3}

Figure 3

Maximum likelihood trees of the all non-coding - ncs (A) and all coding genes - cds (B) data sets. Bootstrap support is given adjacent to the nodes. C. Tree distance (RF) pairwise matrix between all six schemes analyzed. D. Mean bootstrap support of all six schemes analyzed.

A

$\mathrm{C}$

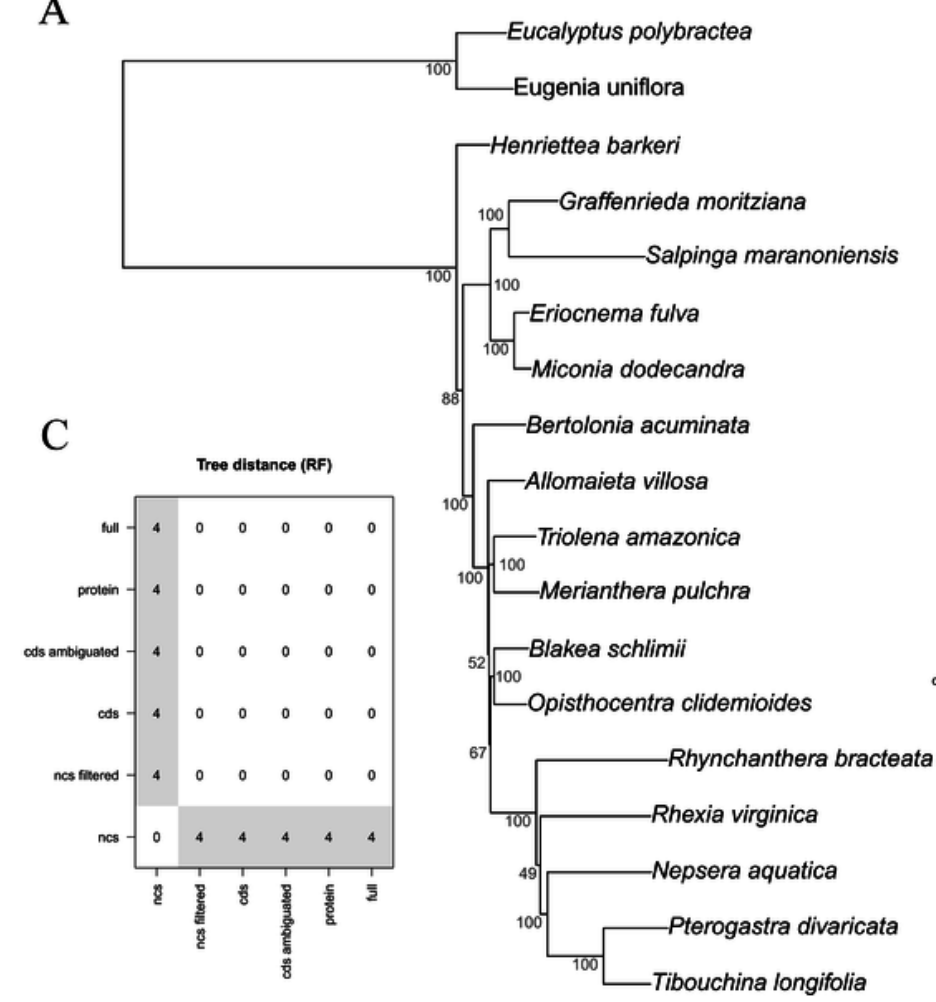

Non-coding all (ncs)

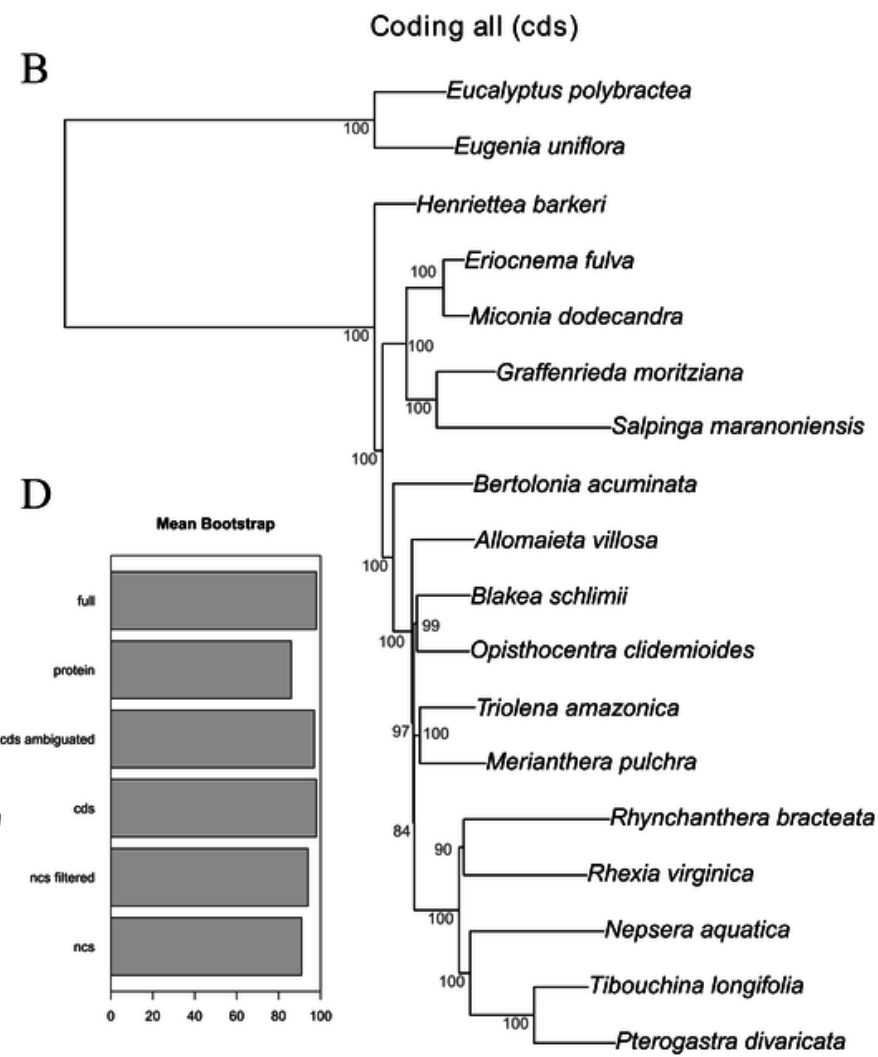




\section{Table 1 (on next page)}

Table 1

Vou cher information and GenBank accessions of the chloroplast sequenced in the Melastomataceae. Informal clades are quoted. 


\begin{tabular}{|c|c|c|c|c|}
\hline Species & Tribe / "clade" & Genbank & Voucher & Herbarium \\
\hline Allomaieta villosa (Gleason) Lozano & Cyphostyleae & KX826819 & David, H. 2188 & HUA, NY \\
\hline Bertolonia acuminata Gardner & Bertolonieae & KX826820 & Goldenberg, R. 810 & NY, UPCB \\
\hline Blakea schlimii (Naudin) Triana & Blakeeae & KX826821 & $\begin{array}{l}\text { Michelangeli, F.A. } \\
1227\end{array}$ & NY \\
\hline Eriocnema fulva Naudin & "Eriocnema" & KX826822 & Almeda, F. 8416 & CAS \\
\hline Graffenrieda moritziana Triana & Merianieae & KX826823 & $\begin{array}{l}\text { Michelangeli, F.A. } \\
832\end{array}$ & NY \\
\hline Henriettea barkeri (Urb. \& Ekman) Alain & Henrietteeae & KX826824 & Ionta, G. 2029 & FLAS \\
\hline Merianthera pulchra Kuhlm. & "Cambessedesia" & KX826825 & Goldenberg, R. 1153 & NY, UPCB \\
\hline Miconia dodecandra Cogn. & Miconieae & KX826826 & $\begin{array}{l}\text { Michelangeli, F.A. } \\
758\end{array}$ & NY \\
\hline Nepsera aquatica (Aubl.) Naudin & "Marcetia" & KX826827 & $\begin{array}{l}\text { Michelangeli, F.A. } \\
1998\end{array}$ & NY \\
\hline Opisthocentra clidemioides Hook. f. & Unplaced & KX826828 & Caddah, M.K. 578 & NY, UPCB \\
\hline Pterogastra divaricata (Bonpl.) Naudin & Melastomeae & KX826829 & $\begin{array}{l}\text { Michelangeli, F.A. } \\
540\end{array}$ & NY \\
\hline Rhexia virginica $\mathrm{L}$. & Rhexieae & KX826830 & $\begin{array}{l}\text { Michelangeli, F.A. } \\
1448\end{array}$ & NY \\
\hline Rhynchanthera bracteata Triana & Microlicieae & KX826831 & Zenteno, F. 8801 & NY \\
\hline Salpinga maranoniensis Wurdack & Merianieae & KX826832 & Clark, J.L. 13577 & UNA \\
\hline Tibouchina longifolia (Vahl) Baill. & Melastomeae & KX826833 & Majure, L. 4277 & FLAS \\
\hline Triolena amazonica (Pilg.) Wurdack & "Triolena" & KX826834 & $\begin{array}{l}\text { Michelangeli, F.A. } \\
1366\end{array}$ & NY \\
\hline
\end{tabular}




\section{Table 2 (on next page)}

Table 2

Comparison of plastid genome size and GC content across different regions in the 16 Melastomataceae species. Length (bp, total \%); GC (GC content \%). LSC (long single copy region); SSC (small single copy region); IR (inverted repeat); Full (full plastome). 


\begin{tabular}{|c|c|c|c|c|c|c|c|c|c|}
\hline \multirow{2}{*}{ Species } & \multirow{2}{*}{$\begin{array}{c}\text { Coverage } \\
\text { (mean) }\end{array}$} & \multicolumn{2}{|c|}{ LSC } & \multicolumn{2}{|c|}{ SSC } & \multicolumn{2}{|c|}{ IR } & \multicolumn{2}{|c|}{ Full } \\
\hline & & $\mathrm{bp}$ & GC & $\mathrm{bp}$ & GC & $\mathrm{bp}$ & GC & $\mathrm{bp}$ & GC \\
\hline Allomaieta villosa & 278 & 85915 & 0.347 & 16975 & 0.306 & 26781 & 0.425 & 156452 & 0.369 \\
\hline Bertolonia acuminata & 189 & 85571 & 0.347 & 17008 & 0.308 & 26733 & 0.425 & 156045 & 0.370 \\
\hline Blakea schlimii & 170 & 85370 & 0.349 & 16998 & 0.308 & 26747 & 0.425 & 155862 & 0.370 \\
\hline Eriocnema fulva & 42 & 85431 & 0.348 & 16953 & 0.308 & 26805 & 0.425 & 155994 & 0.370 \\
\hline Graffenrieda moritziana & 683 & 85341 & 0.347 & 16924 & 0.309 & 26734 & 0.425 & 155733 & 0.370 \\
\hline Henriettea barkeri & 130 & 85991 & 0.347 & 17036 & 0.306 & 26750 & 0.425 & 156527 & 0.369 \\
\hline Merianthera pulchra & 56 & 85621 & 0.348 & 17001 & 0.307 & 26773 & 0.424 & 156168 & 0.370 \\
\hline Miconia dodecandra & 318 & 86609 & 0.348 & 16999 & 0.310 & 26804 & 0.425 & 157216 & 0.370 \\
\hline Nepsera aquatica & 705 & 84644 & 0.348 & 17066 & 0.310 & 26700 & 0.426 & 155110 & 0.371 \\
\hline Opisthocentra clidemioides & 100 & 85866 & 0.348 & 16942 & 0.309 & 26772 & 0.425 & 156352 & 0.370 \\
\hline Pterogastra divaricata & 184 & 84718 & 0.351 & 17156 & 0.312 & 26537 & 0.425 & 154948 & 0.372 \\
\hline Rhexia virginica & 683 & 84459 & 0.351 & 16924 & 0.311 & 26626 & 0.425 & 154635 & 0.372 \\
\hline Rhynchanthera bracteata & 304 & 85093 & 0.347 & 16729 & 0.307 & 26643 & 0.426 & 155108 & 0.370 \\
\hline Salpinga maranoniensis & 537 & 85128 & 0.353 & 16653 & 0.317 & 25765 & 0.428 & 153311 & 0.374 \\
\hline Tibouchina longifolia & 195 & 86297 & 0.349 & 17124 & 0.311 & 26684 & 0.425 & 156789 & 0.371 \\
\hline Triolena amazonica & 48 & 86200 & 0.347 & 16970 & 0.307 & 26741 & 0.425 & 156652 & 0.369 \\
\hline
\end{tabular}

1 


\section{Table 3(on next page)}

Table 3

Comparison of length and GC content across different sequence classes in the plastome of the 16 Melastomataceae species. Length (bp, total \%); GC (GC content \%). 


\begin{tabular}{lcccccccccc}
\hline \multirow{2}{*}{ Species } & \multicolumn{2}{c}{ Protein-coding } & tRNA & \multicolumn{3}{c}{ rRNA } & \multicolumn{3}{c}{ Intron } & \multicolumn{3}{c}{ Intergenic } \\
& $\mathrm{bp}$ & $\mathrm{GC}$ & $\mathrm{bp}$ & $\mathrm{GC}$ & $\mathrm{bp}$ & $\mathrm{GC}$ & $\mathrm{bp}$ & $\mathrm{GC}$ & $\mathrm{bp}$ & $\mathrm{GC}$ \\
\hline Allomaieta villosa & 80826 & 0.374 & 3348 & 0.497 & 9050 & 0.425 & 20553 & 0.347 & 42675 & 0.316 \\
Bertolonia acuminata & 80670 & 0.375 & 3356 & 0.497 & 9050 & 0.425 & 20437 & 0.347 & 42532 & 0.316 \\
Blakea schlimii & 80742 & 0.375 & 3348 & 0.498 & 9050 & 0.425 & 20541 & 0.347 & 42181 & 0.319 \\
Eriocnema fulva & 80628 & 0.375 & 3354 & 0.497 & 9050 & 0.425 & 20540 & 0.347 & 42422 & 0.318 \\
Graffenrieda moritziana & 80286 & 0.375 & 3349 & 0.497 & 9050 & 0.425 & 19691 & 0.347 & 43357 & 0.317 \\
Henriettea barkeri & 80781 & 0.374 & 3363 & 0.495 & 9050 & 0.425 & 20571 & 0.347 & 42762 & 0.315 \\
Merianthera pulchra & 80751 & 0.375 & 3364 & 0.498 & 9050 & 0.425 & 20478 & 0.347 & 42525 & 0.318 \\
Miconia dodecandra & 80586 & 0.376 & 3354 & 0.498 & 9050 & 0.425 & 20548 & 0.347 & 43678 & 0.317 \\
Nepsera aquatica & 80646 & 0.375 & 3370 & 0.496 & 9050 & 0.425 & 20619 & 0.347 & 41425 & 0.318 \\
Opisthocentra clidemioides & 80643 & 0.376 & 3360 & 0.496 & 9050 & 0.425 & 20641 & 0.347 & 42658 & 0.317 \\
Pterogastra divaricata & 80427 & 0.377 & 3339 & 0.498 & 9050 & 0.425 & 19911 & 0.347 & 42221 & 0.318 \\
Rhexia virginica & 80466 & 0.377 & 3353 & 0.496 & 9050 & 0.425 & 20260 & 0.347 & 41506 & 0.319 \\
Rhynchanthera bracteata & 80415 & 0.375 & 3241 & 0.502 & 9048 & 0.425 & 20538 & 0.347 & 41866 & 0.317 \\
Salpinga maranoniensis & 79326 & 0.376 & 3349 & 0.500 & 9050 & 0.425 & 18991 & 0.347 & 42595 & 0.326 \\
Tibouchina longifolia & 80682 & 0.377 & 3348 & 0.497 & 9050 & 0.425 & 20666 & 0.347 & 43043 & 0.317 \\
Triolena amazonica & 80619 & 0.375 & 3337 & 0.496 & 9050 & 0.425 & 20476 & 0.347 & 43170 & 0.316 \\
\hline
\end{tabular}




\section{Table 4 (on next page)}

Table 4

Comparison of plastid genome size of one Melastomataceae species (Miconia dodecandra) with eight other Myrtales. Protein-coding, tRNA and rRNA (number of genes); LSC (long single copy region, length in bp), SSC (small single copy region, length in bp), IR (inverted repeat, length in bp) and Full (length in bp). 


\begin{tabular}{llccccccc}
\hline Family & Species & Coding & tRNA & rRNA & LSC & SSC & IR & Full \\
\hline Melastomataceae & Miconia dodecandra & 84 & 37 & 8 & 86609 & 16999 & 26804 & 157216 \\
Myrtaceae & Allosyncarpia ternata & 84 & 37 & 8 & 88218 & 18571 & 26402 & 159563 \\
Myrtaceae & Angophora costata & 84 & 37 & 8 & 88769 & 18773 & 26392 & 160326 \\
Myrtaceae & Corymbia gummifera & 84 & 37 & 8 & 88310 & 17197 & 27603 & 160713 \\
Myrtaceae & Eucalyptus polybractea & 84 & 37 & 8 & 88944 & 18530 & 26397 & 160268 \\
Myrtaceae & Eugenia uniflora & 84 & 37 & 8 & 87459 & 18318 & 26334 & 158445 \\
Lythraceae & Lagerstroemia fauriei & 84 & 37 & 8 & 83923 & 16933 & 25792 & 152440 \\
Onagraceae & Oenothera grandiflora & 84 & 38 & 8 & 89862 & 19035 & 28824 & 166545 \\
Myrtaceae & Stockwellia quadrifida & 84 & 37 & 8 & 88247 & 18544 & 26385 & 159561 \\
\hline
\end{tabular}

1 


\section{Table 5 (on next page)}

Table 5

Summary statistics for the top 10 introns and intergenic spacers with suitable size for PCR amplification. Markers are ranked by phylogenetic information based on a weighed mean of relative values of number of variable sites (weight $=1$ ), mean bootstrap (weight $=2$ ) and distance to the full plastid tree (weight=3). PIS = parsimony informative sites; Tree distance $=$ RF distance. 


\begin{tabular}{lccccccc}
\hline Marker & Bases & $\begin{array}{c}\text { Aligned } \\
\text { (bp) }\end{array}$ & Variable sites & PIS & $\begin{array}{c}\text { DNA } \\
\text { distance } \\
\text { (mean) }\end{array}$ & $\begin{array}{c}\text { Tree } \\
\text { distance }\end{array}$ & $\begin{array}{c}\text { Bootstrap } \\
\text { (mean) }\end{array}$ \\
\hline 1. trnS-trnG spacer & $780[628,884]$ & 1125 & $438(38.9 \%)$ & $128(11.4 \%)$ & 0.104 & 4 & 82 \\
2. ndhF-rpl32 spacer & $898[849,965]$ & 1266 & $507(40 \%)$ & $171(13.5 \%)$ & 0.114 & 6 & 71 \\
3. trnG intron & $762[743,790]$ & 846 & $236(27.9 \%)$ & $76(9 \%)$ & 0.059 & 4 & 75 \\
4. ndhC-trnV spacer & $734[504,821]$ & 991 & $330(33.3 \%)$ & $98(9.9 \%)$ & 0.081 & 4 & 63 \\
5. ndhA intron & $1016[939,1045]$ & 1127 & $250(22.2 \%)$ & $74(6.6 \%)$ & 0.046 & 4 & 64 \\
6. trnG-atpA spacer & $641[550,750]$ & 895 & $353(39.4 \%)$ & $136(15.2 \%)$ & 0.114 & 6 & 65 \\
7. atpH-atpI spacer & $898[638,980]$ & 1178 & $323(27.4 \%)$ & $92(7.8 \%)$ & 0.062 & 8 & 76 \\
8. psbE-petL spacer & $1058[570,1165]$ & 1396 & $381(27.3 \%)$ & $132(9.5 \%)$ & 0.068 & 8 & 70 \\
9. petA-psbJ spacer & $736[420,944]$ & 1062 & $285(26.8 \%)$ & $90(8.5 \%)$ & 0.076 & 8 & 76 \\
10. trnE-trnT spacer & $842[478,1029]$ & 1345 & $406(30.2 \%)$ & $121(9 \%)$ & 0.089 & 8 & 63 \\
\hline
\end{tabular}

1 


\section{Table 6(on next page)}

Table 6

Primer pair sequences for the indentified top 5 highly informative markers across the 16 plastomes of Melastomataceae. 


\begin{tabular}{lllc}
\hline Marker & Primer forward $\left(5^{\prime}-3^{\prime}\right)$ & Primer reverse $\left(5^{\prime}-3^{\prime}\right)$ & $\mathrm{T}_{\mathrm{a}}\left({ }^{\circ} \mathrm{C}\right)$ \\
\hline 1. $t r n S-$ trn $G$ spacer & CACTCAGCCATCTCTCCCAA & ACCCGCTACAATGCCATTATTG & 55 \\
2. $n d h F-r p l 32$ spacer & AGGAAAGGACCACATACGTCG & TCCTTGCTCATTGATTTTGATCCA & 55 \\
3. $t r n G$ intron & GGTCCCTCGGATTTGCTTCA & GAACCCGCATCGTTAGCTTG & 55 \\
4. $n d h C$ - $t r n V$ spacer & AGATGAACTCCTAGGGAATGTGA & CCGAGAAGGTCTACGGTTCG & 55 \\
5. $n d h A$ intron & CGCTAGTCCAGAACCGTACA & ACCCCATGATTGGTTGATTAGTGA & 55 \\
\hline
\end{tabular}

1 